\title{
Propuesta de un tomógrafo portatil de pocas vistas utilizando haces divergentes
}

\author{
Mariella Berrocal Tito, Hidmer Laulate Melgarejo y Raúl Carita Montero \\ Facultad de Ciencias Físicas, Universidad Nacional Mayor de San Marcos, Ciudad Universitaria. Av. \\ Venezuela s/n, Lima 14, Perú
}

Recibido 24 agosto 2010 - Aceptado 29 setiembre 2010

\begin{abstract}
En este trabajo presentamos un modelo para la construcción de un tomógrafo portátil de pocas vistas. Se considera: (1) un tomógrafo de forma circular con $80 \mathrm{~cm}$ de diámetro, con imágenes reconstruídas circulares de $20 \mathrm{~cm}$ de diámetro. (2) El tomógrafo tiene 6 vistas con 36 conos por vista. Los 216 detectores están fijos sobre la superficie interna del tomógrafo. (3) Las fuentes puntuales están en posiciones fijas. Para la reconstrucción de imagen utilizamos una discretización que considera los caminos de la radiación entre la fuente y el detector, la intersección de estos caminos forman una discretización denominada base natural. El algoritmo de reconstrucción empleado es el r-ART, basado en utilizar la distancia de Bregman como un término de regularización. La distancia de Bregman es construída con una función convexa que tiene un parámetro $r=0.1$. Presentamos ejemplos de imágenes reconstruidas. Las imágenes reconstruidas simulan la cabeza de un niño con una anomalía. Los datos para la reconstrucción fueron obtenidos mediante el código Monte Carlo de N Partículas, MCNP.

Palabras claves: Tomografía; reconstrucción; problemas inversos; algoritmo; distancia de Bregman.
\end{abstract}

\section{Proposal of a portable few view tomograph with divergent beams}

In this paper we present a model for building a portable computed tomography (CT) scanner of few views. We consider: (1) a circular tomograph of $80 \mathrm{~cm}$ diameter, with a reconstructed image of 20 $\mathrm{cm}$ diameter. (2)The scanner has 6 views with 36 cones per view. The 216 detectors on the inner surface scanner are fixed. (3)The point sources are in fixed position. For image reconstrucction, we use a discretization through radiation paths between sources and detectors, the intersection of these pathways form a natural base discretization. The reconstruction algorithm is the r-ART, based in Bregman's distance as a regularization term. Bregman's distance is constructed with a convex function with a parameter $r=0.1$. Reconstructed images examples are presented. The reconstructed images show a child head with an anomaly. The reconstruction data was obtained using the Monte Carlo N Particle ,MCNP, code.

Keywords: Tomography; reconstruction, inverse problems, algorithm, Bregman's distance.

El desarrollo de la tomografía computarizada permite obtener imágenes de las estructuras internas del cuerpo de manera no invasiva. Con el paso de los años la técnica ha avanzado a pasos agigantados, pero aún hoy en día continúa siendo un campo de gran interés para la investigación y desarrollo.

Las imágenes de tomografía convencional son reconstruidas a partir de un gran número de medidas de los rayos $\mathrm{X}$ que pasan a través del paciente. Las imágenes resultantes son mapas tomográficos de los coeficientes de atenuación lineal[1]. La evolución de la tomogra- fía computarizada, es descrita generalmente en términos de generaciones que reflejan además su desarrollo histórico. Así tenemos, la primera generación de haces paralelos, donde la geometría es técnicamente simple, la fuente y el detector se mueven de manera lineal en un barrido angular, para luego volver y hacer un nuevo barrido, así hasta completar los 180 grados, el tiempo necesario para completar un corte era de $5 \mathrm{~min}$ [2]. Este aparato fue inventado por Sir Godfrey Hounsfield y lo hizo merecedor del Premio Nóbel de Medicina en 1979[3]. La segunda generación tenía el haz en forma

*mabet99@yahoo.com 
de abanico y múltiples detectores dispuestos en forma de serie lineal, el tiempo de barrido se redujo aproximadamente a $30 \mathrm{~s}$, presentaba movimiento de rotación y traslación, el algoritmo de reconstrucción es ligeramente más complicado. La tercera generación también presentaba los haces en forma de abanico, pero los detectores también rotaban, todo el sistema giraba 360 grados a través de su isocentro. Debido a esto el tiempo de procesamiento y obtención de la imagen se redujo a 1s. La cuarta generación continúa con el haz en forma de abanico, pero presenta los detectores fijos en un círculo alrededor del paciente, así solo la fuente da rayos $\mathrm{X}$ gira. La quinta generación (tomografía computarizada por haz de electrones), cuenta con un cañón emisor de electrones, este haz se deflecta por magnetismo incidiendo sobre láminas de tungsteno, generando en esta interacción los rayos $\mathrm{X}$ que giran sin que lo haga ninguna parte del sistema. El procesamiento de los datos se dan en aproximadamente $50 \mathrm{~ms}[4]$. El requerimiento de tiempos más cortos ha llevado al desarrollo de la tomografía computarizada helicoidal, en donde la fuente de rayos $\mathrm{X}$ y los detectores se posicionan sobre anillos deslizantes, el haz de rayos $\mathrm{X}$ traza un dibujo en forma de hélice sobre la superficie del paciente, los datos se procesan por ciclos, llevando a tiempos de exploración de 0.7 y 1 s por ciclo[5-6].

La tomografía de pocas vista implica la disminución de la exposición a radiaciones y el tiempo de procesamiento de la información.

\section{Problema directo}

La tomografía computarizada, CT, es un proceso para obtener una imagen bidimensional de una sección transversal de un cuerpo, partiendo del conocimiento de las interacciones de los rayos $\mathrm{X}$ con el medio a través del cual se propaga; esta interacción está sustentada en base a la ecuación de la transferencia radiativa[7-9] y del transporte de partículas, que es una expresión matemática de la conservación de la energía radiante[10].

Un problema directo en tomografía computariza$\mathrm{da}[11]$ consiste en conocer la geometría, la intensidad de la radiación que entra en el medio (se consideran fuentes externas de radiación) y los coeficientes de atenuación, con lo cual puede ser calculada la intensidad de salida del medio, mediante la siguiente ecuación

$$
\frac{d I}{d s}+\sigma(x, y) I=0
$$

donde $I(x, y)$ es la intensidad de radiación, $\sigma(x, y)$ es el coeficiente de atenuación y $d s$ es el diferencial de camino.

Resolviendo la ecuación (1), se obtiene la intensidad de la radiación en la salida del medio, la que es medida por los detectores como

$$
I_{D}=I_{S} \exp \left[\int_{\text {ray }} \sigma(x, y) d s\right]
$$

o mediante

$$
\ln \frac{I_{S}}{I_{D}}=\int_{\text {ray }} \sigma(x, y) d s
$$

siendo $I_{S}$ la intensidad de la fuente e $I_{D}$ la intensidad registrada por el detector.

En nuestro caso para encontrar esta medida de los detectores es necesario particionar el dominio, esto es lograr una geometría adecuada para procesar los datos.

\section{Partición del dominio}

La partición del dominio es la segmentación de un determinado espacio, para manipular convenientemente la información que se pueda obtener a partir de ella. En este trabajo utilizaremos la base natural, que está determinada por los caminos por donde pasa la radiación en un medio determinado, estos caminos forman conos y la intersección de conos forman polígonos, cada polígono es denominado elemento de área[12,13].

La ecuación (3) la aproximamos como sumatoria debido a la partición

$$
\ln \frac{I_{S}}{I_{D}}=\sum_{e \in\{j, k\}}^{E} \sigma_{e} a_{e}=h_{j, k}
$$

donde $j$ se denomina vista, $j=1,2, \ldots, J$, siendo $J$ el número máximo de vistas. Asimismo $k$ indica el número de cono, $k=1,2, \ldots, K$, siendo $K$ el número máximo de conos equivalente al número total de medidas de los detectores. La intersección de los caminos de la radiación es la intersección de los conos que generan los polígonos denominados elementos de área. Tenemos que $e$ es un elemento de área, con $e=1,2, \ldots, E$, donde $E$ es el número total de elementos de área. A cada elemento de área va asociado un coeficiente de atenuación $\sigma_{e} \mathrm{y}$ un área $a_{e}$ definido. Finalmente $h_{j, k}$ está vinculado a las intensidades medidas en la vista $j$ y en el cono $k$.

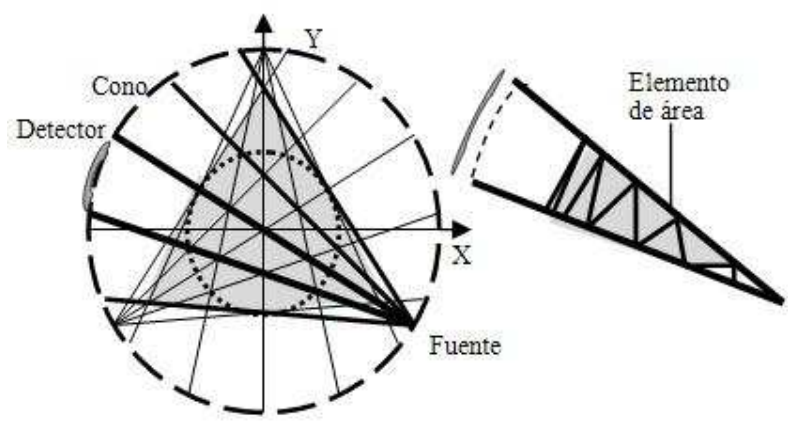

Figura 1: Partición del dominio haces divergentes. 
En la Figura 1 se muestra esta partición de dominio, para 3 vistas y 4 conos para cada vista. Los polígonos encierran elementos de área cada uno asociado a un valor de coeficiente de atenuación.

\section{Problema inverso}

El problema inverso consiste en determinar las causas de un fenómeno físico basadas en la observación de sus efectos[14], así para obtener imágenes en tomografía computarizada es necesario conocer la intensidad de la fuente de rayos X, y la intensidad colectada por los detectores después de la interacción con el medio[15]. La solución tras un algoritmo de reconstrucción nos lleva a los coeficientes de atenuación, así obtenemos una imagen representativa de medio.

Definimos la matriz $A$ con dimensiones $(K, E)$, donde las filas están relacionadas a un cono, y cada columna a un elemento de área, así una fila $k$ contiene toda la información de los elementos de área que forman el cono $k$. $\vec{\sigma}$ es el vector columna formado por todas las incógnitas, $\vec{h}=A \vec{\sigma}$ es el vector columna relacionado a las medidas de los detectores. La representación matricial está dado por

$$
\left(\begin{array}{c}
h_{1} \\
\vdots \\
h_{K}
\end{array}\right)=\left(\begin{array}{ccc}
a_{11} & \cdots & a_{1 E} \\
\vdots & \vdots & \vdots \\
a_{K 1} & \cdots & a_{K E}
\end{array}\right)\left(\begin{array}{c}
\sigma_{1} \\
\vdots \\
\sigma_{K}
\end{array}\right) \text {. }
$$

Definimos la función error como la diferencia entre la medida experimental del detector y el calculado

$$
\vec{F}=A \vec{\sigma}-\vec{h} \text {. }
$$

Construímos la lagrangiana $L$ utilizando la distancia de Bregman $D$, la cual es calculada por una función relacionada con la entropía de Havrda-Charvat cuando $r=0.1$, de modo que

$$
L\left(\vec{\sigma}, \vec{\sigma}^{0}\right)=D\left(\vec{\sigma}, \vec{\sigma}^{0}\right)+\lambda^{T} \vec{F}
$$

donde $\lambda$ son los multiplicadores de Lagrange y la distancia de Bregman es determinada por

$$
D\left(\vec{\sigma}, \vec{\sigma}^{0}\right)=-\sum_{e=1}^{E}\left(\sigma_{e}-\sigma_{e}^{0}-\sigma_{e} \ln \frac{\sigma_{e}}{\sigma_{e}^{0}}\right),
$$

en ambas ecuaciones el superíndice 0 , indica un valor a priori. El problema inverso fue resuelto empleando la lagrangiana de la Ec.(7) y el algoritmo r-ART, descrito en los trabajos[16,17].

Así para estimar $\sigma$, la ecuación (7) es resuelto como un problema de optimización, para esto debe de cumplir con dos requisitos:

1. $\nabla_{\sigma} L=\nabla_{\sigma} D+\nabla_{\sigma}\left(\lambda^{T} F\right)=0$, de donde se obtiene

$$
\sigma^{r-1}=\sigma^{0, r-1}-\frac{r-1}{r} A^{T} \lambda .
$$

2. $\nabla_{\lambda} L=\nabla_{\lambda} D+F+\left(\nabla_{\lambda} F^{T}\right) \lambda=0$, de donde obtenemos

$$
\left(\nabla_{\lambda} \sigma^{T}\right)_{k, e}=\frac{\partial \sigma_{e}}{\partial \lambda_{k}}=-\frac{1}{r} \sigma_{e}^{2-r} A_{k, e} \quad .
$$

Considerando la convergencia en la determinación de $\lambda$ para un cono determinado, éste es usado para obtener un coeficiente de atenuación estimado.

\section{Propuesta}

La motivación para el desarrollo de este tomógrafo computarizado portátil de pocas vistas se fundamenta básicamente en que la intensidad de radiación absorbida por los pacientes sea la más posible, especialmente en niños. El riesgo de cáncer en niños debido a la exposición a la radiación es alrededor de 2 a 3 veces más alto que en adultos, esto se debe a que ellos tienen una expectativa de vida más larga y sus órganos son más radiosensibles[18-22].

El tomógrafo computarizado de pocas vistas que proponemos está constituído por 6 fuentes de rayos $\mathrm{X}$ con una energía de $60 \mathrm{KeV}$, la disposición de los detectores es simétrica a lo largo de una circunferencia de $80 \mathrm{~cm}$ de diámetro, en ubicaciones fijas. Son necesarios 36 detectores localizándose frente a cada fuente, teniendo en total 216 detectores (Fig. 2). La región para la obtención de las imágenes y que son cubiertas por la partición del dominio esta dado por una circunferencia de $20 \mathrm{~cm}$ de diámetro que representa nuestra región de interés. Como protección radiológica utilizamos un blindaje exterior de plomo de $3 \mathrm{~mm}$ de espesor. En la tabla 1 presentamos los coeficientes medios de atenuación y las densidades de los materiales simulados.

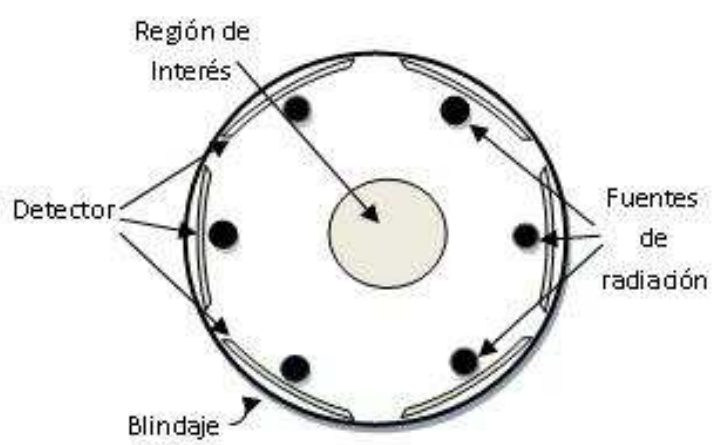

Figura 2: Esquema del tomógrafo propuesto. 


\begin{tabular}{lll}
\hline Materiales & $\begin{array}{l}\text { Coef. de atenua- } \\
\text { ción lineal a } 60 \\
\mathrm{KeV}\left(\mathrm{cm}^{-1}\right)\end{array}$ & $\begin{array}{l}\text { Densidad } \\
\left(\mathrm{g} / \mathrm{cm}^{3}\right)\end{array}$ \\
\hline Aire & 0,00023 & 0,013 \\
Cerebro & 0,210 & 1,04 \\
Hueso & 0,416 & 1,6 \\
\hline
\end{tabular}

Tabla 1: Coeficientes de atenuación y densidad de aire, cerebro y hueso, usados en este trabajo[19].

\section{Código MCNP}

El código de Monte Carlo de N partículas, MCNP, es un software que utiliza el método Monte Carlo para la simulación de la interacción de la radiación con la materia.

El usuario crea un archivo de entrada que es subsecuentemente leído por el programa MCNP. Este archivo contiene información sobre el problema en áreas tales como: la especificación de la geometría, la descripción del material, localización y características de la fuente, tipo de respuestas y cualquier técnica de reducción de variancia usado para mejorar la eficiencia.

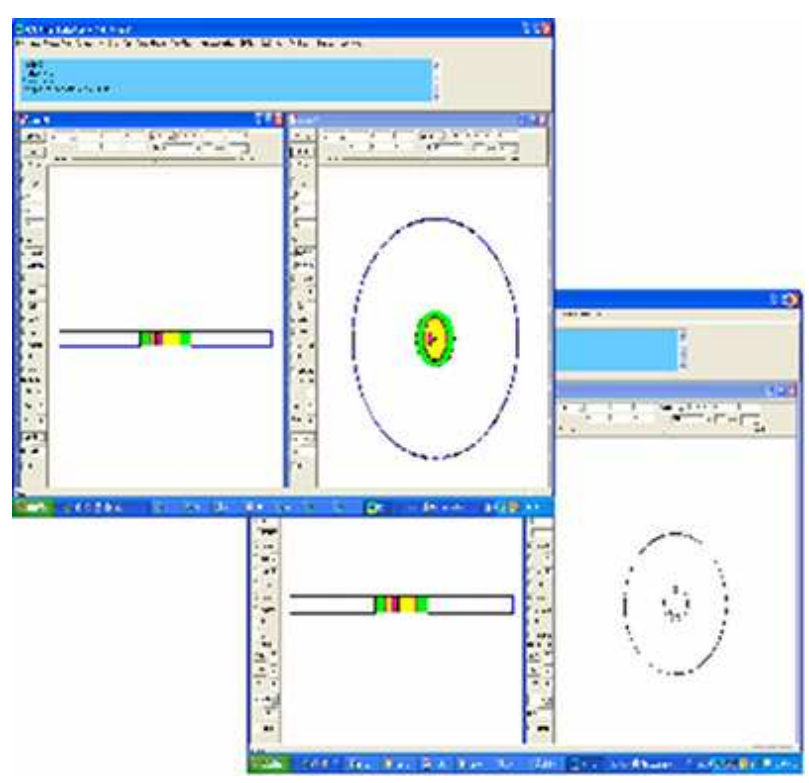

Figura 3: Especificación de geometría en el programa MCNP.

La geometría utilizada se muestra en la Fig. 3, en la región de interés se logra apreciar una imagen representativa de una cabeza (corte de un cráneo) que contiene una anomalía en el cerebro, rodeado por aire. Se utilizan los materiales de la Tabla I para lo cual es necesario sus composiciones de manera porcentual y sus respectivas densidades. Las 6 fuentes de fotones son ubicadas dentro de los blindajes de plomo de manera simétrica como se indicó anteriormente.

\section{Resultados y discusión}

Utilizando la partición del dominio y el algoritmo r-ART, propuesto en el problema directo. Presentamos el primer caso simulado comenzando por la Fig.4a que muestra la imagen original a reconstruir, la elipse simula el cráneo donde se observa una anomalía que podría ser un carcinoma, un meningioma o un hematoma. Por simplicidad consideramos que los coeficiente de atenuación del hueso y la anomalía sean $\sigma_{h}=0.416 \mathrm{~cm}^{-1}$ mostrados en rojo, en la región interna del cráneo consideramos $\sigma_{c}=0.210 \mathrm{~cm}^{-1}$ mostrados como verde claro y en la región exterior al cráneo consideramos el coeficiente del aire $\sigma_{a}=0.00023 \mathrm{~cm}^{-1}$ de color azul. El detalle de los valores que representan los colores está en la franaj de colores de la derecha de los gráficos.

La Fig.4b presenta la imagen reconstruída a partir de los datos generados por el problema directo con un ruido aleatorio de $0.1 \%$, la tonalidad amarilla de los huesos y la anomalía muestra cierta dispersión aleatoria que puede ser originada por la misma naturaleza de la elipse tomada como geometría básica. Pero, esta dispersión originada aparentemente por la elipse se reduce drásticamente cuando la imagen es reconstruída usando el código MCNP como se muestra en la Fig.4c. El detalle del craneo mejora notablemente y mantiene su identidad. La anomalía mantiene su forma y el constraste respecto al tejido cerebral que la rodea permite una mejor identificación aunque de color menos rojizo que la del craneo, lo cual puede ser un dato referencial importante para el diagnóstico clínico de la anomalía.

Para la ejecución de los programas, se empleó una Laptop, con procesador Intel Core 2 Duo de $2.0 \mathrm{GHz}$. y $320 \mathrm{~GB}$ de HD. La versión del MATLAB es 7.04. El tiempo computacional para la reconstrucción empleado datos del problema directo fue de $135.906 \mathrm{~s}$ y el tiempo computacional para datos generados usando el código MCNP fue de $461.813 \mathrm{~s}$.

\section{Conclusiones}

Los resultados presentados muestran que el procesamiento de los datos de los coeficientes de atenuación lineal usando el método de Monte Carlo presenta una mejor imagen de las anomalías discriminándolas de los tejidos que ofrecen también un mayor coeficiente de atenuación lineal de los rayos $\mathrm{X}$ que la atraviesan como los huesos del cráneo. Es posible que para imágenes más complejas se presente algunos problemas en la recons- 
trucción. Sin embargo sabemos por los trabajos anteriores[16] que una discretización más fina de la malla mejora la calidad de la imagen reconstruída.

Esta propuesta de construcción de un tomógrafo portátil de pocas vistas, conlleva a la disminución de la dosis absorbida por el paciente debido al número de
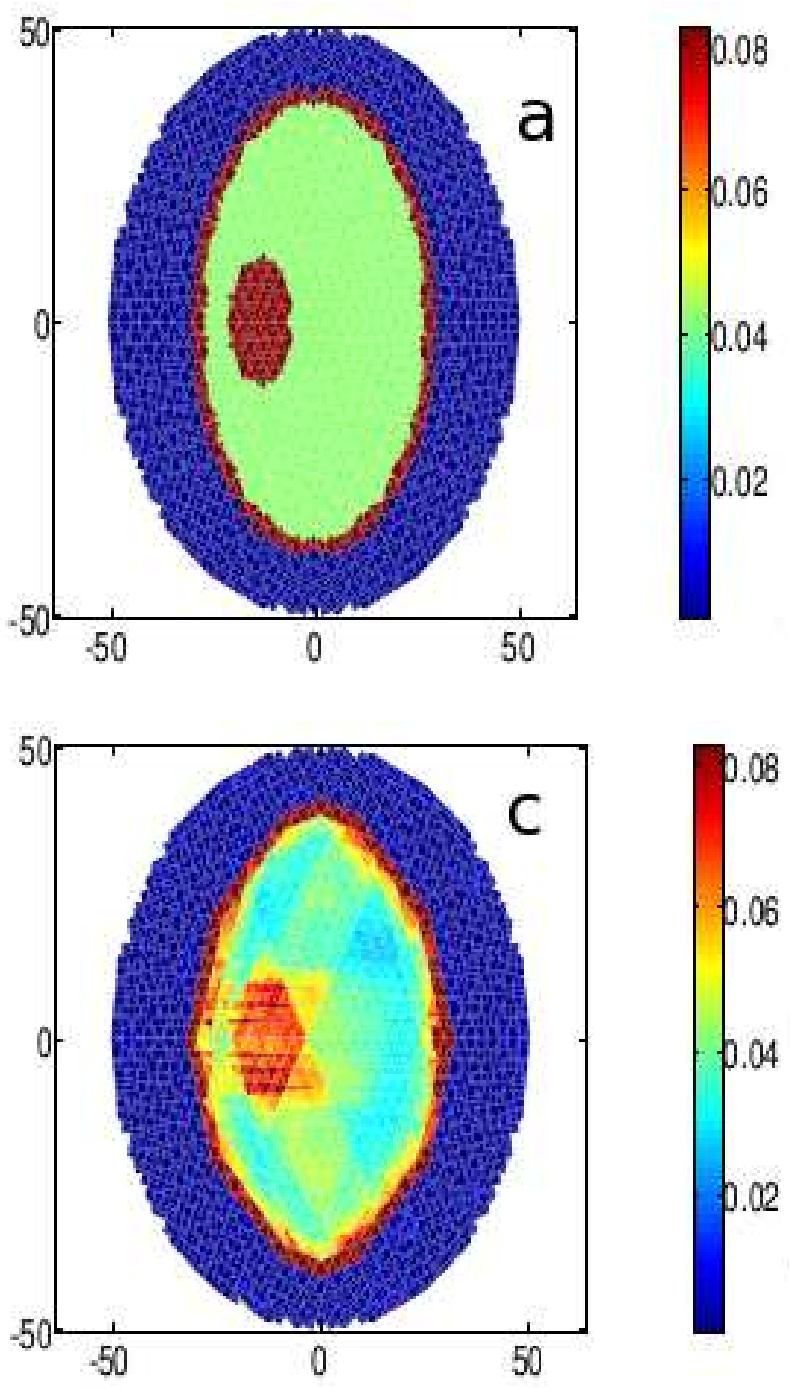

vistas, mucho menor que en los tomógrafos modernos. Por lo cual creemos que es importante continuar este trabajo.

Una mayor discretización de la malla esta prevista en nuestros trabajos futuros.

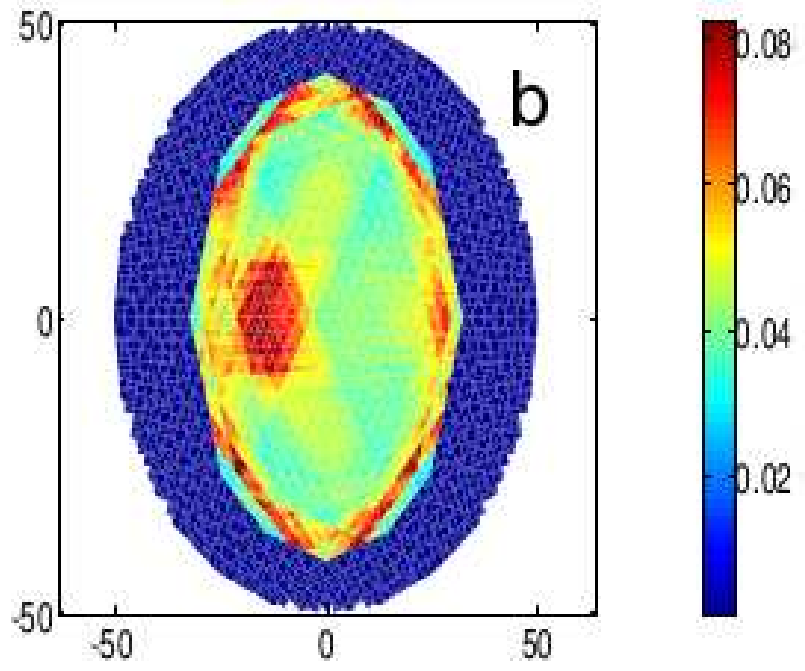

Figura 4: a) Imagen de anomalía en cerebro. b) Imagen reconstruída de 6 vistas en un método numérico. c) Imagen reconstruída de 6 vistas a partir de datos generados el código MCNP. Consideramos una unidad de longitud igual a $5 \mathrm{~cm}$.

\section{Referencias}

[1] J. Bronzino, The Biomedical Engineering Handbook, CRC Press, LLC/IEEE Press, Florida (1995).

[2] F.J. Cabrero, Imagen radiológica: Principios físicos e instrumentación, Masson, Barcelona (2006).

[3] O.E. Bosch, Sir Godfrey Newbold Hounsfield y la tomografía computada, su contribución a la medicina moderna, Rev. Chil. Radiol. 10, 183 (2004).

[4] S.C. Bushong, Computed Tomography: Essentials of Medical Imaging, McGraw Hill, New York, (2000).

[5] G. Konrad, H. Smith, H. Jorgen, P. Holger, A. Da- 
vid, The Encyclopaedia of Medical Imaging, Vol. 1, Taylor \& Francis, London (1998).

[6] J.T. Bushberg, The Essential Physics of Medical Imaging, 2nd Ed., Lippencott Williams \& Wilkins, Philadelphia (2002).

[7] M.J. Berrocal, N.C. Roberty, A.J. Silva Neto, J. Bravo, Inverse radiative transfer problems in twodimensional participating media, Inverse Problems in Science and Engineering 12, 103 (2004).

[8] R.F. Carita, N.C. Roberty, A.J. Silva Neto, Reconstruction of a combination of the absorption and scattering coefficients with a discrete ordinates method consistent with the source-detector system, Inverse Problems in Science And Engineering 12, 81 (2004).

[9] M.J. Berrocal, R.F. Carita, C.E. Velásquez, El método de Monte Carlo y la solución de la ecuación de transferencia radiativa, Rev. Inv. Fis. 13, 101302751 (2010).

[10] S. Chandrasekhar, Radiative Transfer, Dover, New York (1960).

[11] R.F. Carita, N.C. Roberty, A.J. Silva Neto, $A b-$ sorption Coefficient Estimation in Two-Dimensional Media Using a Base Constructed with Divergent Beams, Inverse Problems in Engineering 9, 587 (2001).

[12] R.F. Carita, N.C. Roberty, A.J. Silva, Natural Base Construction for Absorption Coefficient Estimation in Heterogeneous Participating Media with Divergent Beams: Theory and Practice, 3rd International Conference on Inverse Problems in Engineering, June 13-18, Port Ludlow, WA, USA (1999).

[13] M.J. Berrocal, R.F. Carita, Algoritmos ART para a estimativa do coeficiente de absorção com feixes divergentes usando certas funções de Bergman, Tend. Mat. Apl. Comput. 8, 181 (2007).

[14] C. Groetsch, Inverse Problems. Activities for Undergraduates, The Mathematical Association of America, USA (1999).
[15] T.M. Buzug, Computed Tomography: From Photon Statistics to Modern Cone-Beam CT, Springer Science \& Business Media, Berlin (2009).

[16] M.J. Berrocal, R.F. Carita, A.J. Silva Neto, Reconstrucción de imágenes en tomógrafos de pocas vistas, Rev. Inv. Fis. 11, 42 (2008).

[17] C. Velásquez, M.J. Berrocal, R.F. Carita, Algoritmos r-ART para reconstrucción de imágenes en tomógrafos de pocas vistas usando datos generados por el metodo de Monte Carlo, V Congreso Latinoamericano de Física Medica, Cuzco, Asociación Latino Americana de Física Medica, mayo (2010).

[18] D.J. Benner, C.D. Elliston, E.J. Hall, W.E. Berdon, Estimated risks of radiation-induced fatal cancer from pediatric CT, Am. J. Roentgnol. 176, 289 (2001).

[19] C. Hollingsworth, D.P. Frush, M. Cross, J. Lucaya, Helical CT of the Body: A survey of Techniques Used for Pediatric Patients, Am. J. Roentgnol. 180, 401 (2003).

[20] A. Paterson, D.P. Frush, Dose reduction in paediatric MDCT: general principles, Clin. Radiol. Jun. 62, 507 (2007)

[21] F.A. Mettler Jr., P.W. Wiest, J.A. Locken, C.A. Kelsey, CT scanning: patterns of use and dose, J. Radiol. Prot. 20, 353 (2000).

[22] H. Laulate, M.J. Berrocal, R.F. Carita, Propuesta de un tomógrafo portátil de pocas vistas utilizando haces divergentes, $\mathrm{V}$ Congreso Latinoamericano de Física Medica, Cuzco, Asociación Latino Americana de Física Medica, mayo (2010).

[23] G.T. Herman, Fundamentals of Computerized Tomography. Image Reconstruction from Projections, 2nd Ed., Springer, London (1979).

[24] I.M. Solov, Método de Monte Carlo, Ed. Mir, Moscú (1976). 